\title{
Efficacy and safety of olaparib maintenance therapy in platinum-sensitive ovarian cancer patients with BRCA mutations: a meta-analysis on randomized controlled trials
}

This article was published in the following Dove Press journal:

Cancer Management and Research

\author{
Jiao $\mathrm{Ma}^{1}{ }^{1} *$ \\ Hongyong Deng ${ }^{2, *}$ \\ Jiajia $\mathrm{Li}^{1}$ \\ Shaopu $\mathrm{Hu}^{3}$ \\ Yanping Yang ${ }^{3}$ \\ Sheng Liu' \\ Xianghui Han'
}

'Institute of Chinese Traditional Surgery, Longhua Hospital Affiliated to Shanghai

University of Traditional Chinese

Medicine, Shanghai 200032, People's

Republic of China; ${ }^{2}$ Key Laboratory of

Theory and Therapy of Muscles and

Bones, Ministry of Education, Shanghai

200032, People's Republic of China;

${ }^{3}$ Science and Information Center,

Shanghai University of Traditional

Chinese Medicine, Shanghai 201203,

People's Republic of China

*These authors contributed equally to this work

\begin{abstract}
Background: Olaparib, a potent oral poly (ADP-ribose) polymerase inhibitor, exhibits antitumor activity and prevents the recurrence in advanced ovarian cancer. In this article, we assessed the efficacy and safety of olaparib maintenance therapy on platinum-sensitive ovarian cancer patients with $B R C A$ mutations through a meta-analysis of available randomized controlled trials (RCTs) to provide more evidence for its clinical applications.

Methods: We searched PubMed, Embase, Wanfang, CNKI, Web of Science, Cochrane Library, and VIP databases from 1 August 2018 to identify RCTs and finally included four RCTs (seven articles) with 567 eligible participants beyond the participants, interventions, comparisons, outcomes, and study design regulation. The outcomes of olaparib efficacy including progression-free survival (PFS) and overall survival (OS) were measured by $\mathrm{HR}$ and $95 \% \mathrm{CI}$, while the quality of life was evaluated by calculating the combination of $P$-value. Seven common adverse events were tested by risk ratio and $95 \% \mathrm{CI}$ as the outcomes of olaparib safety. These data were analyzed, and the forest figures were produced using Review Manager 5.3.

Results: Compared with other interventions (ie, placebo or chemotherapy drugs), olaparib significantly prolonged PFS ( $\mathrm{HR}=0.31,95 \% \mathrm{CI}=0.15-0.62)$ and slightly improved $\mathrm{OS}$ (HR $=0.75,95 \% \mathrm{CI}=0.56-0.99)$, but did not influence the quality of life $(P=0.058)$ in the patients with platinum-sensitive $B R C A$-mutated ovarian cancer. Additionally, the toxicity profile of olaparib involved anemia, fatigue, vomiting, diarrhea, and nausea with grade 1-2. Conclusion: This meta-analysis suggests that olaparib maintenance therapy is effective and well-tolerated for the patients with platinum-sensitive BRCA-mutated ovarian cancer. More updated RCTs and long-term follow-up should be conducted to compare and analyze the efficacy and toxicity of olaparib at different doses in ovarian cancer patients.
\end{abstract}

Keywords: olaparib, ovarian cancer, efficacy, adverse event, meta-analysis

\section{Introduction}

Ovarian cancer is the seventh most common cancer for women overall worldwide. ${ }^{1}$ In the UK, there were about 7,400 new cases of ovarian cancer in 2014, and it remains the leading cause of death which accounted for $5 \%$ of all cancer deaths. ${ }^{2}$ In the US, there would be approximately 11.6 new cases per 100,000 women and over 6.7 deaths per 100,000 women from the disease in 2015. And in 2018, there will be estimated 22,240 new cases and 14,070 deaths of ovarian cancer. ${ }^{3}$ In China, there 
were 52,100 women diagnosed with ovarian cancer and 14,080 patients died of this cancer in $2015 .{ }^{4}$ Treatments for ovarian cancer including operative therapy, chemotherapy, and targeted therapy are used in clinical practice. ${ }^{5-8}$

Olaparib (also called lynparza) is an oral, potent inhibitor of poly (ADP-ribose) polymerase (PARP), and its chemical name is 4-[3-(4-cyclopropanecarbonylpiperazine-1carbonyl)-4-fluorobenzyl]-2H-phthalazin-1-one. ${ }^{9} \quad$ Olaparib was developed by AstraZeneca Cooperation (London, $\mathrm{OH}$, USA) and used to treat various advanced tumors, including breast, prostate, pancreatic, and ovarian cancers. ${ }^{10-14}$ In 2014, the FDA and the EMA approved olaparib treatment in germline BRCA-mutated advanced ovarian cancer that has received three or more prior lines of chemotherapy. ${ }^{14-18}$ Additionally, several Phase II trials have reported that olaparib maintenance therapy in platinum-sensitive relapsed serous ovarian cancer could diminish the tumor size, prolong progression-free survival (PFS), and improve objective responses rate in patients. ${ }^{14,19,20}$ Meanwhile, it presented an acceptable and manageable tolerability profile and had no worse impact on health-related quality of life. ${ }^{21,22}$

However, most of these trials have a limitation of small sample size and may not accurately evaluate the efficacy and safety of olaparib. The present study included all available randomized controlled trials (RCTs) according to the criteria of participants, interventions, comparisons, outcomes, and study design (PICOS), and then conducted a meta-analysis to estimate the efficacy and safety of oral olaparib maintenance therapy vs placebo or other chemotherapy drugs among the adult patients with platinumsensitive $B R C A$-mutated ovarian cancer. It is expected to provide some evidence on clinical extensive use of olaparib in advanced ovarian cancer.

\section{Methods}

\section{Search strategy}

We systematically searched seven databases which contain PubMed (May 2009 to July 2018), Embase (February 2008 to April 2018), Wanfang (February 2013 to April 2017), CNKI (September 2016 to April 2018), Web of Science (May 2008 to May 2018), Cochrane Library (September 2011 to October 2017), and VIP database (February 2015 to November 2017) up until 1 August 2018 using the following terms: "olaparib OR lynparza OR azd 2,281 OR azd2,281 OR azd221" AND "ovarian tumor OR ovarian cancer OR ovarian carcinoma OR ovarian neoplasm". In addition, we hand-searched the posters and power points from the American Society of Clinical Oncology Annual Meeting and the reports from AstraZeneca Pharmaceuticals (London, OH, USA).

\section{Selection criteria}

According to PICOS criteria, the studies were selected and evaluated independently by two reviewers. The relevant clinical trials on the efficacy and safety of olaparib maintenance therapy for platinum-sensitive ovarian cancer patients with $B R C A$ mutations were included, if they met the following criteria: 1) the trial had an RCT design; 2) the trial enrolled the patients older than 18 years of age with $B R C A$ mutations ovarian cancer, which had received at least two previous courses of platinum-based chemotherapy regimen; 3) the trial compared olaparib with other interventions such as placebo or other chemotherapy drugs; and 4) the trial presented at least one of the following outcomes: a) PFS, b) overall survival (OS), c) quality of life, and d) adverse events. For the trials without complete information, we contacted the authors to obtain the unpublished data. If the authors could not provide the necessary data, the trials were excluded.

\section{Selection of studies}

Two review authors independently screened the titles and abstracts of all the articles, which obtained through the search strategy. Then, we retrieved the full texts and assessed all eligible studies in compliance with the inclusion criteria. A third review author was available to resolve any discrepancies.

\section{Data extraction}

The data were extracted from each included trial using a piloted data extraction sheet by two investigators independently. The extracted data summarized the characteristics of trials (first author, inclusion criteria, interventions, outcomes, and other necessary information). If disagreement occurred, all review authors discussed for consensus.

\section{Assessment of risk of bias}

According to the description of Cochrane Handbook for Systematic Reviews of Interventions 5.3 (Higgins 2011), two review authors independently assessed risk of bias in each included trial. We resolved disagreements by consulting a third review author. We applied the Cochrane "Risk of bias" tool to evaluate the following domains:

1. random sequence generation (selection bias)

2. allocation concealment (selection bias) 
3. blinding of participants, personnel, and outcome assessment (performance bias and detection bias)

4. incomplete outcome data (attrition bias)

5. selective reporting data (reporting bias)

We considered the generation of allocation sequence to be adequate if the trial reported any truly random process (eg, random number table and computer-generated random sequence number). We considered the allocation concealment to be adequate if the trial reported sufficient details about intervention allocation (eg, telephone or central randomization and consecutively numbered sealed opaque envelopes). We considered the blinding study of participants, personnel, and outcome assessors to be adequate if the trial was definitely mentioned the indistinguishability between experimental and control preparations. We considered the incomplete outcome data to be adequate if the trial reported that there were no losses in patients to follow-up and no treatment withdrawals according to the intention-to-treat principle. We considered the selective reporting data to be adequate if the trial showed that all of the study's prespecified outcomes had been reported in the published reports. Additionally, we did not consider the item "free of other bias" in this review.

\section{Measures of efficacy and adverse events}

For time-to-event outcomes (efficacy such as PFS and OS), we calculated the HR between treatment groups with 95\% CI. For dichotomous outcomes (adverse events), we calculated the risk ratio (RR)RR with $95 \%$ CI.

\section{Data analysis}

Quantitative data were imported into Review Manager 5.3 and then analyzed using a fixed-effect model $(P>0.10$ or $\mathrm{I}^{2}<50 \%$ ), if not, using a random-effect model. The inverse variance and Mantel-Haenszel were used for the estimates of typical HR and RR. We planned to do the subgroup analyses by type of intervention groups. We included four RCTs that met our selection criteria and compared olaparib with other drug treatment (eg, placebo, pegylated liposomal doxorubicin [PLD], or paclitaxel plus carboplatin). We used the outcomes of OS, PFS, and adverse events in subgroup analyses.

\section{Results}

\section{Description of studies} Results of the search

A total of 2,063 records were retrieved from seven databases, of which 2,032 were excluded following title and abstract screening. We obtained 31 full-text articles to be potentially eligible for this review. Next, we excluded five articles that were no RCTs, two articles that were Phase I trials, and eight articles with no available data. Second, we excluded two articles (Liu 2014 and Liu 2017) because they aimed at investigating the effect of cediranib in combination with olaparib. Next, we excluded another article (Ledermann 2012) due to the occurrence of the updated articles. Finally, we included seven full-text articles to assess in this review (Kaye 2012; Ledermann 2014; Ledermann 2016 [September]; Ledermann 2016 [November]; Oza 2015; Pujade-Lauraine 2017; Friedlander 2018). ${ }^{19,20,23-29}$ The flowchart of the selection process is shown in Figure 1.

\section{Characteristics of included studies}

Of the four included trials (seven reports), one compared olaparib with PLD (Kaye 2012); one compared olaparib vs paclitaxel plus carboplatin (Oza 2015); while two compared olaparib with placebo (Ledermann 2014; Ledermann 2016 [September]; Ledermann 2016 [November]; PujadeLauraine 2017; Friedlander 2018).

Kaye 2012 was a multicenter, randomized, and openlabel Phase II study. It enrolled 96 patients with histologically or cytologically confirmed $B R C A$-mutated recurrent epithelial ovarian, primary peritoneal, or fallopian tube carcinoma and assigned them to receive olaparib (200 or $400 \mathrm{mg}$ twice daily) or PLD $\left(50 \mathrm{mg} / \mathrm{m}^{2}\right)$. Disease recurred or progressed within 12 months of the most recent platinum-based chemotherapy regimen. The primary outcome was PFS and the secondary outcomes included RECIST (response evaluation criteria in solid tumors)-defined complete or partial response, duration of treatment response, tumor size, OS, safety, tolerability, and health-related quality of life for each treatment group.

The trial reported by Ledermann 2014, 2016 (September) and 2016 (November) was a multicenter, randomized, and double-blind Phase II study. It divided 264 patients (including 136 cases with $B R C A$ mutations and 128 cases with wild-type $B R C A$ ) with recurrent ovarian, fallopian tube cancer, or primary peritoneal cancer into two groups receiving olaparib (400 $\mathrm{mg}$ twice daily) or placebo, respectively. Patients had received two or more previous lines of platinum-based chemotherapy. The primary outcome was PFS and the secondary outcomes were time to progression, objective response rate, disease-control rate, tumor size, OS, disease-related symptoms, health-related quality of life, safety, tolerability, time 


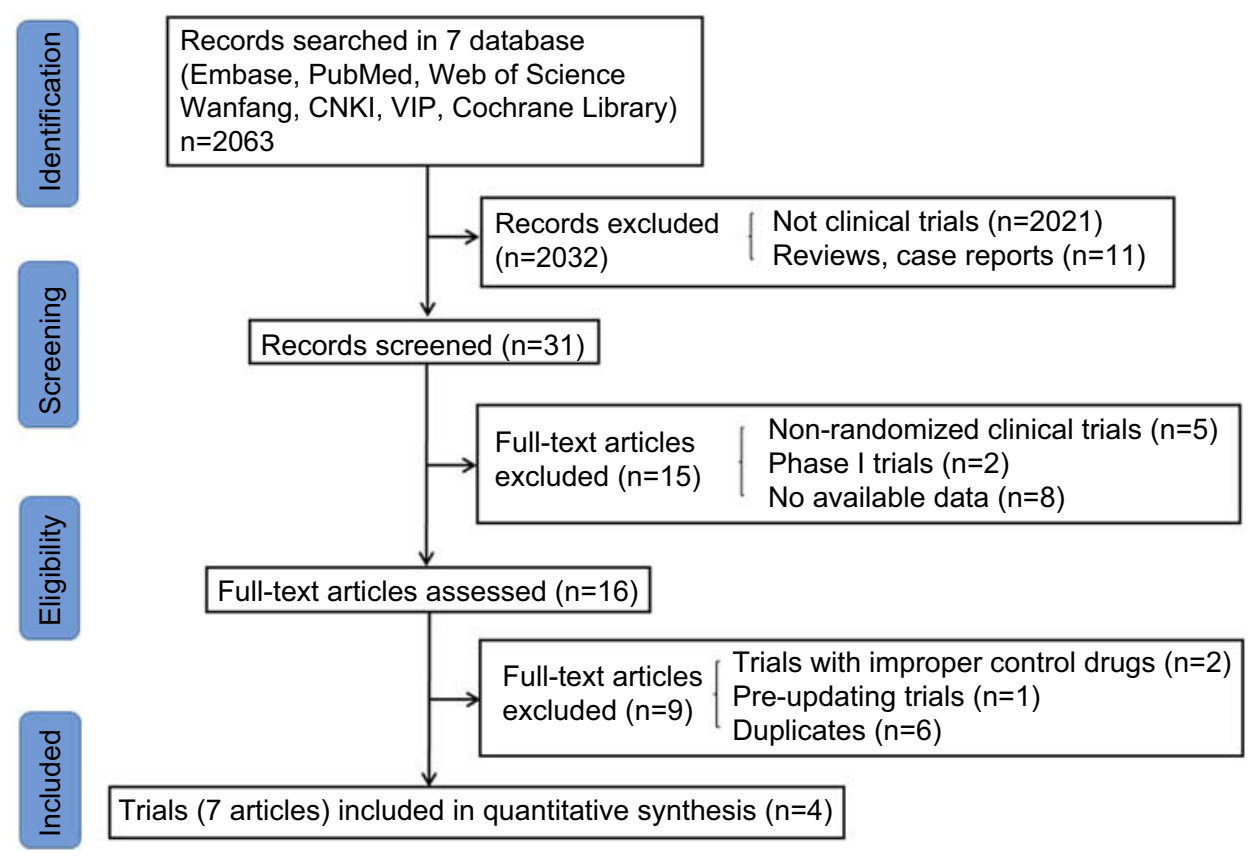

Figure I Flowchart of the number of studies identified and included into the meta-analysis.

to first subsequent therapy or death, time to second subsequent therapy or death, objective response rate, and best overall response.

Oza 2015 conducted a multicenter, open-label Phase II study which enrolled 156 patients (including 41 patients with $B R C A$ mutations) with histologically or cytologically diagnosed platinum-sensitive ovarian cancer, including primary peritoneal and fallopian tube cancers. Patients had received a maximum of three previous lines of platinum-based chemotherapy. These patients were randomized to receive olaparib (200 $\mathrm{mg}$ twice daily) plus paclitaxel $\left(175 \mathrm{mg} / \mathrm{m}^{2}\right.$ ) and carboplatin (AUC $4 \mathrm{mg} / \mathrm{mL}$ per $\mathrm{min}$ ) or paclitaxel $\left(175 \mathrm{mg} / \mathrm{m}^{2}\right)$ and carboplatin (AUC $6 \mathrm{mg} / \mathrm{mL}$ per min). The primary outcome was PFS and the secondary outcomes included OS, the percentage change in tumor size, and the proportion of patients with a RECIST or cancer antigen 125 response.

Pujade-Lauraine 2017 and Friedlander 2018 were both international, multicenter, double-blind, randomized, placebo-controlled, Phase III studies. Two hundred and ninety-four patients with $B R C A$ mutations were histologically confirmed relapsed, high-grade serous ovarian cancer or high-grade endometrioid cancer. Patients had received at least two previous lines of platinum-based chemotherapy. All enrolled patients were divided into two groups which received olaparib (300 $\mathrm{mg}$ twice daily) or placebo, respectively. The primary outcome was PFS and the secondary outcomes included time to first subsequent therapy or death, time to second subsequent therapy or death, time to study treatment discontinuation or death, time to second progression, time to earliest progression or death, investigator assessment of OS, safety, tolerability, and healthrelated quality of life. Further details of the above studies are provided in Table 1.

\section{Risk of bias in included studies}

Figure 2 demonstrates the judgments and specific information on risk of bias in the studies.

\section{Random sequence generation}

Random sequence was adequately generated in all included trials, as they used computer randomization or a random numbers table (Kaye 2012; Ledermann 2014; Ledermann 2016 [September]; Ledermann 2016 [November]; Oza 2015; Pujade-Lauraine 2017; Friedlander 2018).

\section{Allocation concealment}

Allocation was adequately concealed in three trials (six reports) which used the telephone or central randomization for allocation of treatment (Ledermann 2014; Ledermann 2016 [September]; Ledermann 2016 [November]; Oza 2015; Pujade-Lauraine 2017; Friedlander 2018). And the remaining one was at unclear risk of selection bias without mentioning whether the schedule was concealed or not (Kaye 2012). 


\begin{tabular}{|c|c|c|c|}
\hline 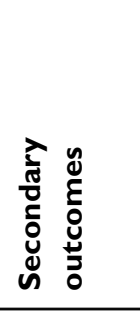 & 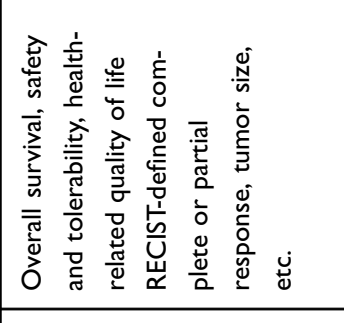 & 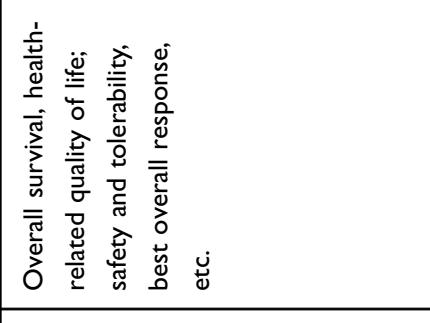 & 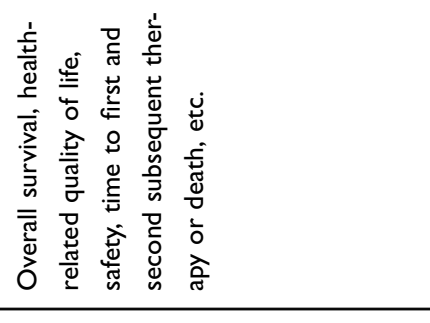 \\
\hline 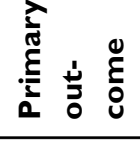 & 奠 & 兽 & 总 \\
\hline 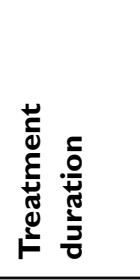 & 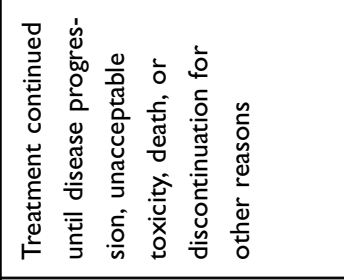 & 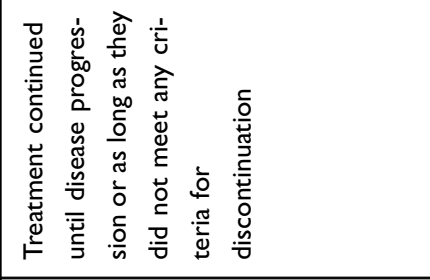 & 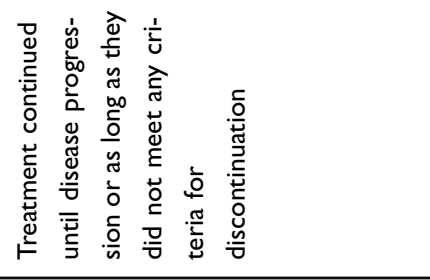 \\
\hline 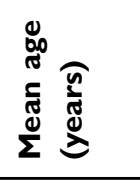 & 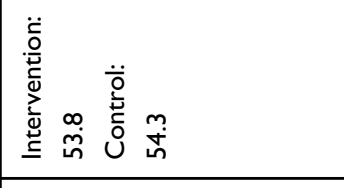 & 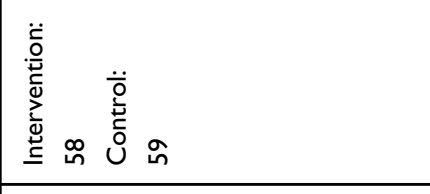 & 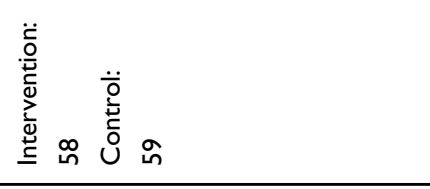 \\
\hline 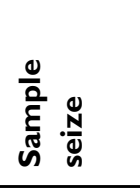 & 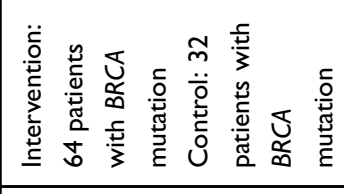 & 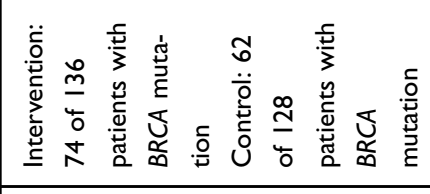 & 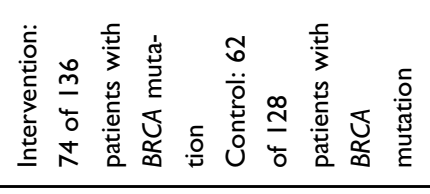 \\
\hline 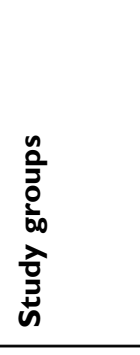 & 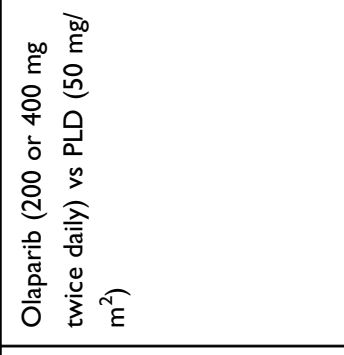 & 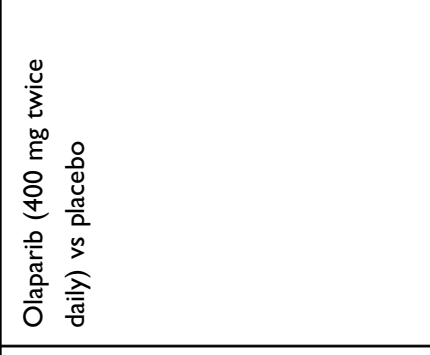 & 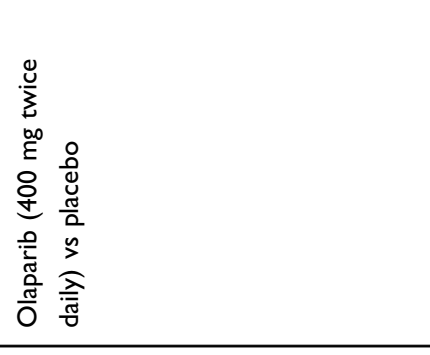 \\
\hline 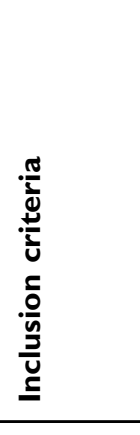 & 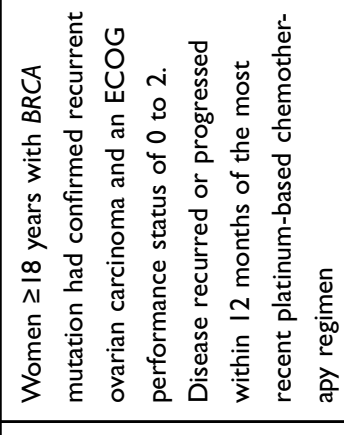 & 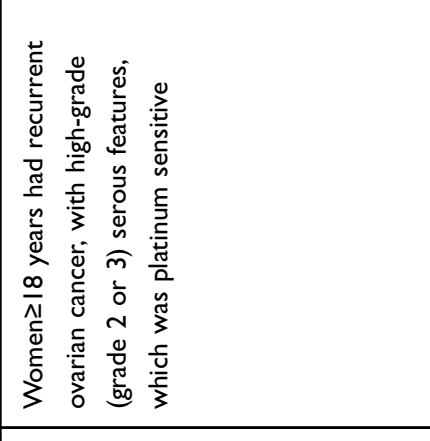 & 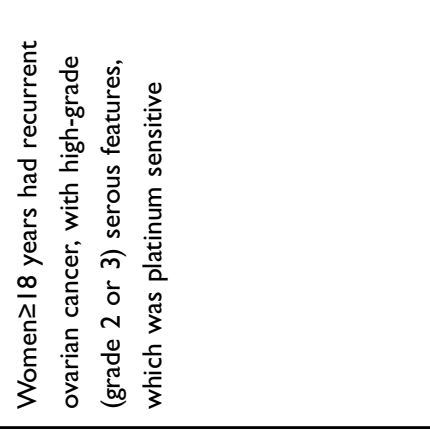 \\
\hline 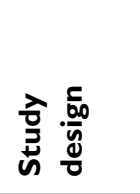 & 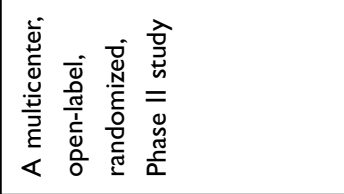 & 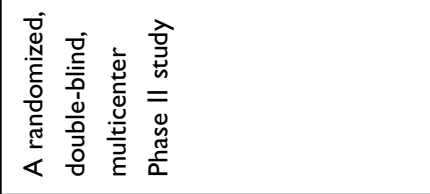 & 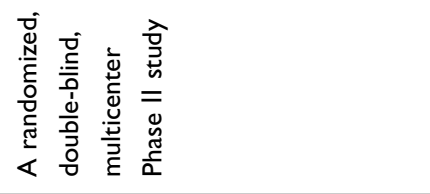 \\
\hline 蒙衰 & 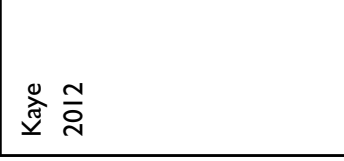 & 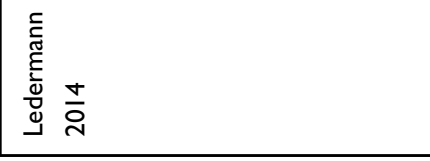 & 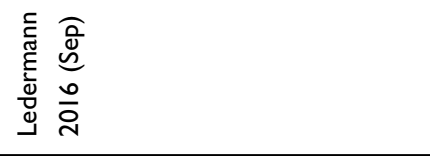 \\
\hline
\end{tabular}




\begin{tabular}{|c|c|c|c|c|c|}
\hline & 气્ّ & 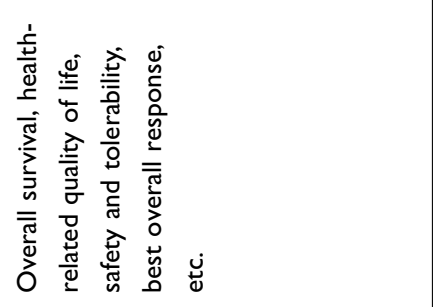 & 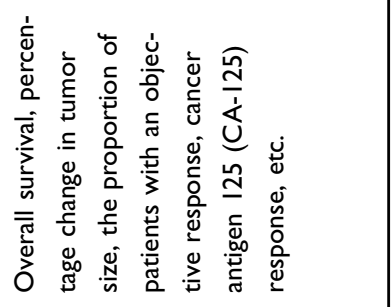 & 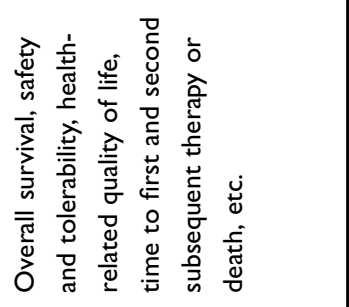 & 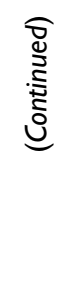 \\
\hline & 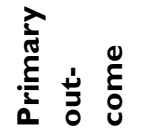 & 㟔 & 씀 & 荝 & \\
\hline & 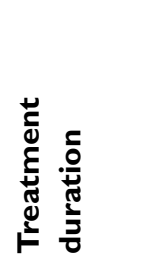 & 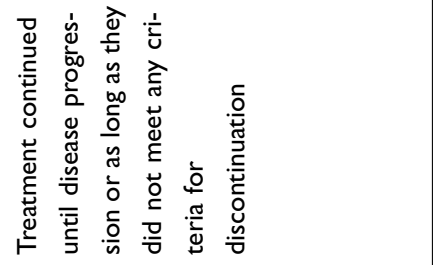 & 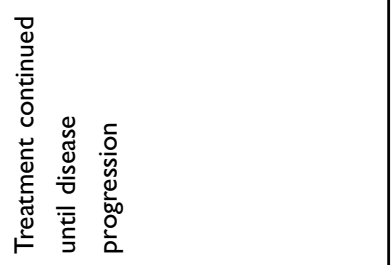 & 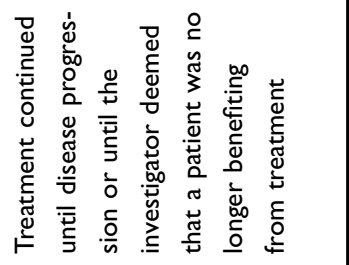 & \\
\hline & 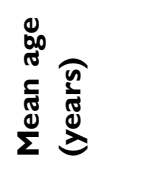 & 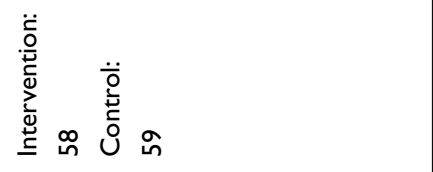 & 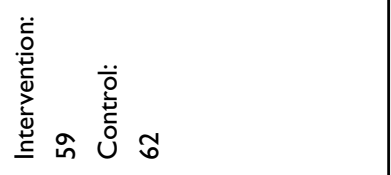 & 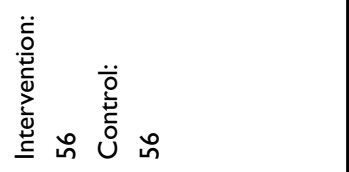 & \\
\hline & 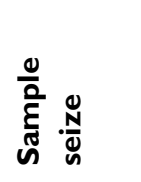 & 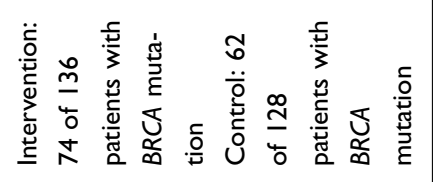 & 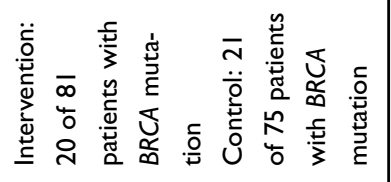 & 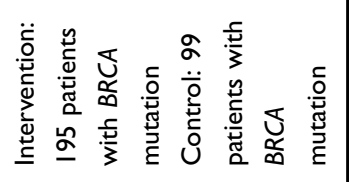 & \\
\hline & 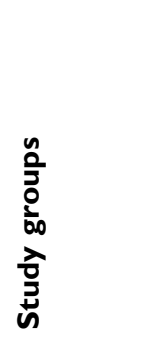 & 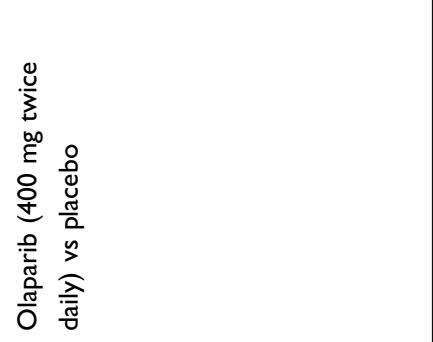 & 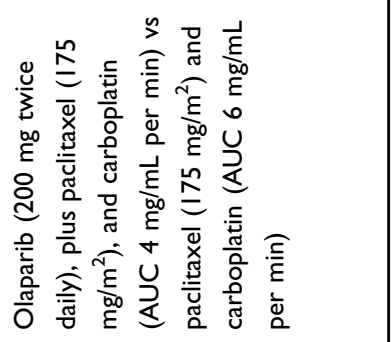 & 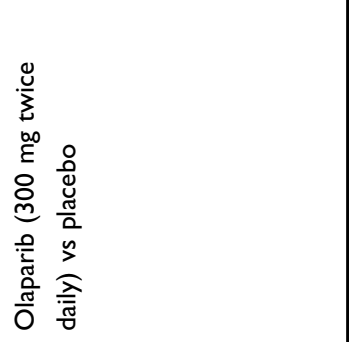 & \\
\hline & 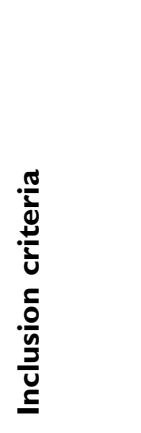 & 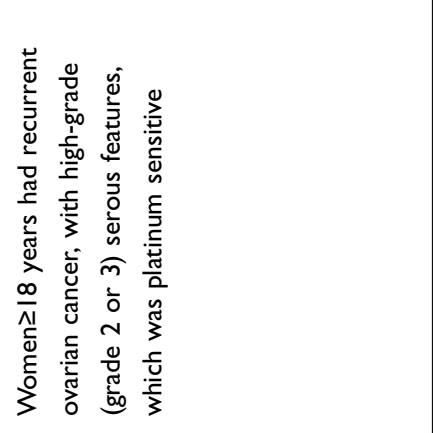 & 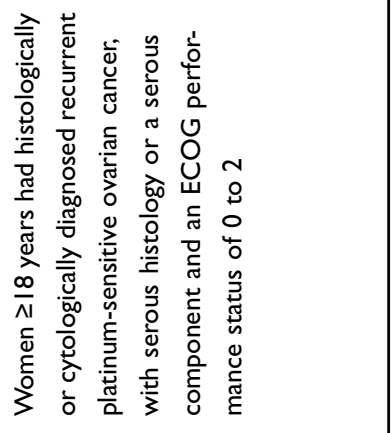 & 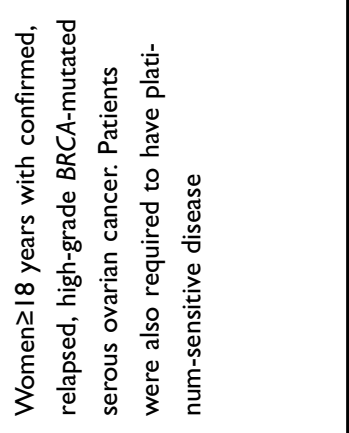 & \\
\hline$\stackrel{0}{\partial}$ & 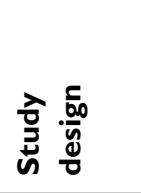 & 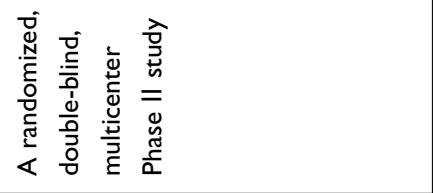 & 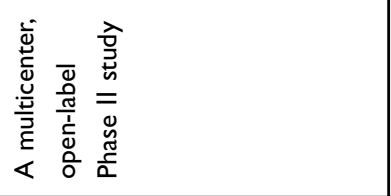 & 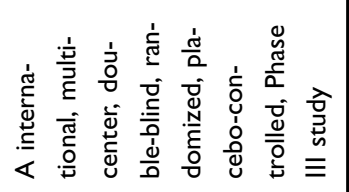 & \\
\hline $\begin{array}{l}\frac{-}{0} \\
\frac{0}{0} \\
\frac{10}{1}\end{array}$ & 岕 & 茍 & Õ $\frac{n}{2}$ & 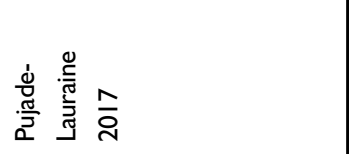 & \\
\hline
\end{tabular}




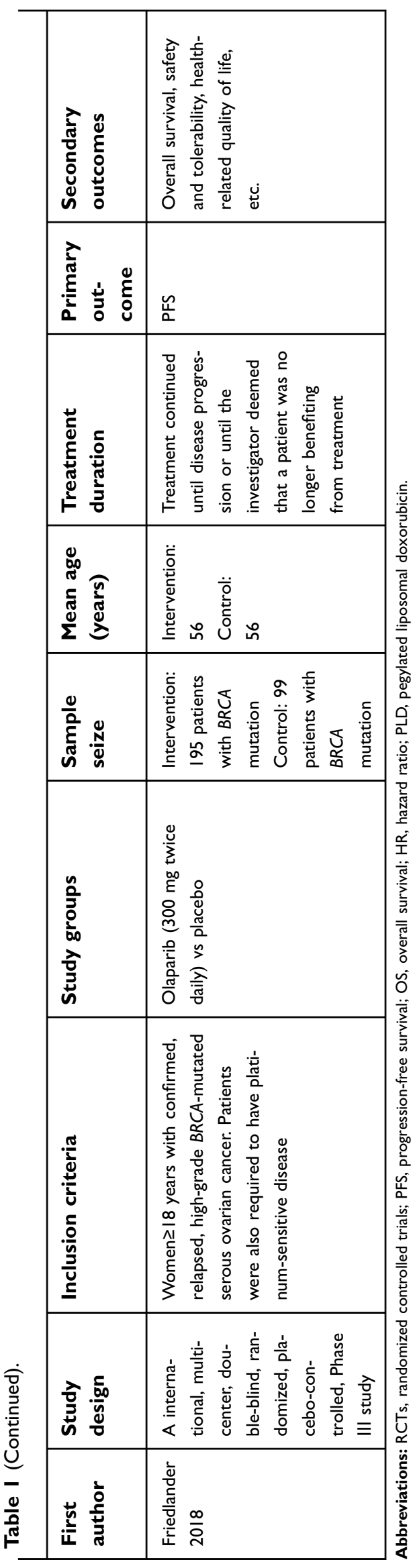

\section{Blinding}

Treatment assignment and outcome were masked for participants, personnel, and outcome assessment in two trials, we considered the risk of performance bias was low for blinding (Ledermann 2014; Ledermann 2016 (Sep); Ledermann 2016 [November]; Pujade-Lauraine 2017; Friedlander 2018). And the other two trials were at high risk of performance bias and detection bias because participants, personnel, and outcome assessors were open-label (Kaye 2012; Oza 2015).

\section{Incomplete outcome data}

Four trials ( 5 reports) had discontinued treatment because of adverse events, death, lost to follow-up and other reasons (Kaye 2012; Ledermann 2016 [September]; Oza 2015; Pujade-Lauraine 2017; Friedlander 2018) and one trial (two reports) lacked the analysis of intention-to-treat basis (Ledermann 2014; Ledermann 2016 [November]). So we judged these trials were at high risk of attrition bias.

\section{Selective reporting}

The pre-specified primary outcomes had been reported. So we judged four trials (5 reports) to be at low risk of outcome reporting bias for our primary outcomes in the published articles (Kaye 2012; Ledermann 2014; Ledermann 2016 [September]; Oza 2015; PujadeLauraine 2017). However, we judged three trials (three reports) at low risk (Kaye 2012; Oza 2015; PujadeLauraine 2017) and one trial (two reports) (Ledermann 2014; Ledermann 2016 [September]) at high risk for all secondary outcomes. Two trials (Ledermann 2016 [November]; Friedlander 2018) only reported the data on quality of life, so we judged them at high risk.

\section{Quantitative synthesis}

\section{Progression-free survival (PFS)}

Four trials reported the outcome of PFS. There were 567 randomly assigned participants, including 353 in olaparib groups and 214 in other intervention groups. These clinical trials evaluated olaparib against placebo or other chemotherapy drugs in platinum-sensitive ovarian cancer patients with BRCA mutations. The treatment duration continued until disease progression, unacceptable toxicity, or death in patients. The quality of evidence for this outcome was high. The pooled data revealed that PFS was significantly prolonged when olaparib was used as opposed to placebo or other chemotherapy drugs (HR $=0.31,95 \%$ CI $=0.15-0.62$, random-effect model, 

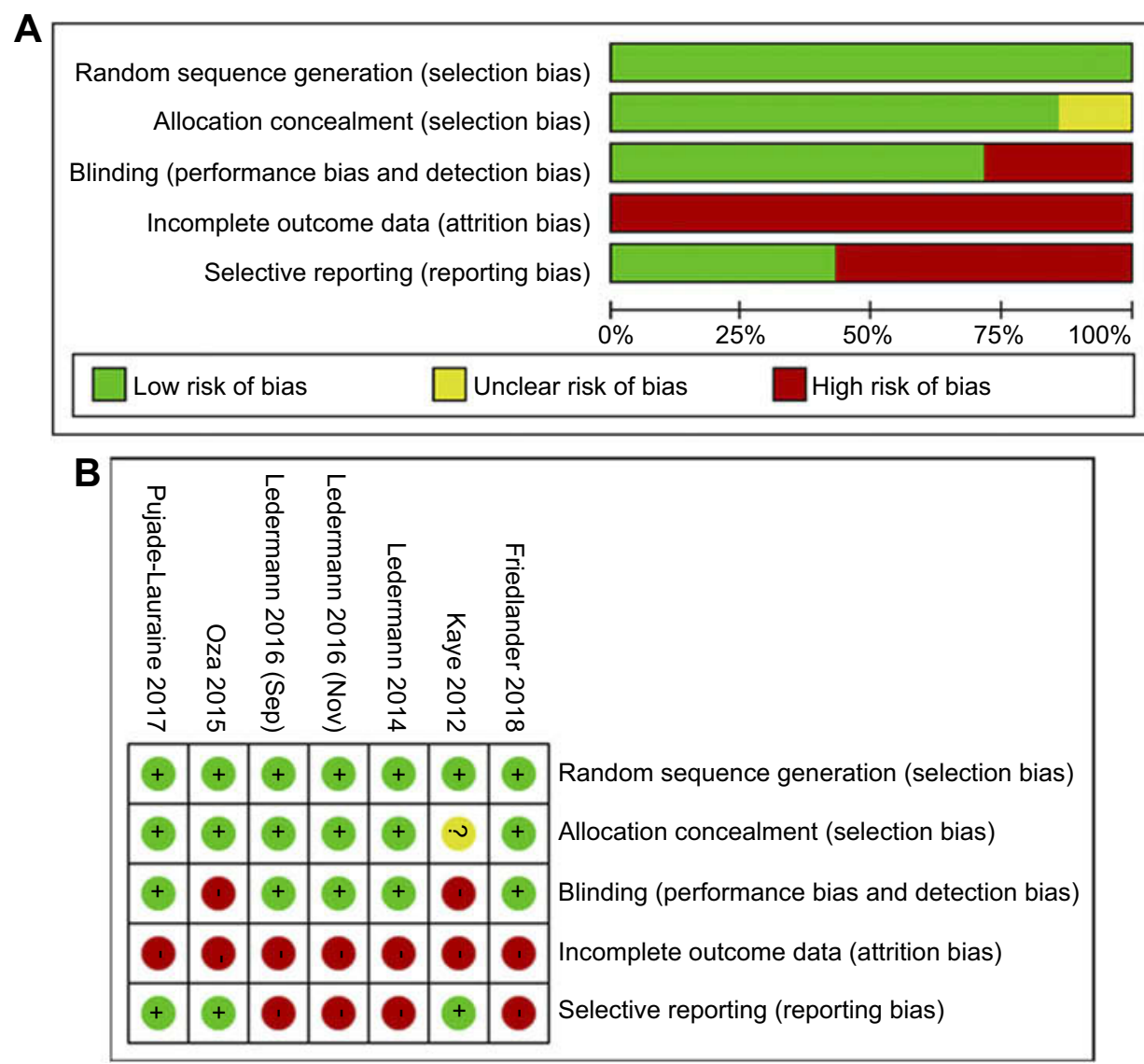

Figure 2 (A) Risk of bias graph: review authors' judgments about each risk of bias item presented as percentages across all included studies. (B) Risk of bias summary: review authors' judgments about each risk of bias item for each included study.

Figure 3). The heterogeneity was statistically significant $\left(P=0.0004, \mathrm{I}^{2}=84 \%\right)$.

In two trials (Ledermann 2014, Pujade-Lauraine 2017) comparing olaparib with placebo, the HR of median PFS was $0.23(95 \% \quad \mathrm{CI}=0.17$ to $0.31, n=430, \quad P<0.00001)$ for 269 patients in olaparib groups and 161 patients in placebo groups. One trial (Oza 2015) compared olaparib plus paclitaxel and carboplatin versus paclitaxel and carboplatin in ovarian cancer treatment. PFS was significantly longer in olaparib plus paclitaxel and carboplatin group (median 12.2 months) than in paclitaxel and carboplatin group (median 9.6 months) $(\mathrm{HR}=0.21,95 \% \mathrm{CI}=0.08$ to $0.55, \mathrm{n}=41, P=0.002)$. Additionally, a meta-analysis of the one comparative trial (Kaye 2012) between olaparib $200 \mathrm{mg}$ or $400 \mathrm{mg}$ and PLD $\left(50 \mathrm{mg} / \mathrm{m}^{2}\right)$ showed no significant difference in median PFS $(\mathrm{HR}=0.88,95 \% \mathrm{CI}=0.50$ to $1.56, n=96, P=0.66)$.

\section{Overall survival (OS)}

Four trials reported the data concerning OS in ovarian cancer with 353 patients in olaparib groups compared to 214 patients in other intervention groups. OS was slightly increased in ovarian cancer patients with olaparib contrast to placebo or other chemotherapy $(\mathrm{HR}=0.75,95 \% \mathrm{CI}=0.56$ to $0.99, \mathrm{n}=567, P=0.05$; Figure 3 ). A fixed-effect model for heterogeneity revealed this result $\left(P=0.54, \mathrm{I}^{2}=0 \%\right)$. We assessed the quality of this evidence to be moderate.

In terms of olaparib and placebo treatment for platinum-sensitive ovarian cancer patients with $B R C A$ mutations, one trial of Ledermann 2016 (September) reported the 5-year survival and one trial of Pujade-Lauraine 2017 did not mention the data cutoff for survival in both groups. The OS in these trials was significantly higher in olaparib groups compared with placebo groups $(\mathrm{HR}=0.69 ; 95 \%$ $\mathrm{CI}=0.51-0.95, \mathrm{n}=430, P=0.02)$. Moreover, in comparison of olaparib and other chemotherapy drugs for ovarian cancer, two trials showed no evidence of a change in OS (Oza 2015, HR=1.28, 95\% CI=0.39-4.20, $\mathrm{n}=41, P=0.68$; Kaye $2012, \mathrm{HR}=1.01,95 \% \mathrm{CI}=0.44-2.32, \mathrm{n}=96, P=0.98)$.

\section{Quality of life}

Three trials reported quality of life in olaparib-treated ovarian cancer patients with $B R C A$ mutations $(\mathrm{n}=526)$. 


\section{PFS}

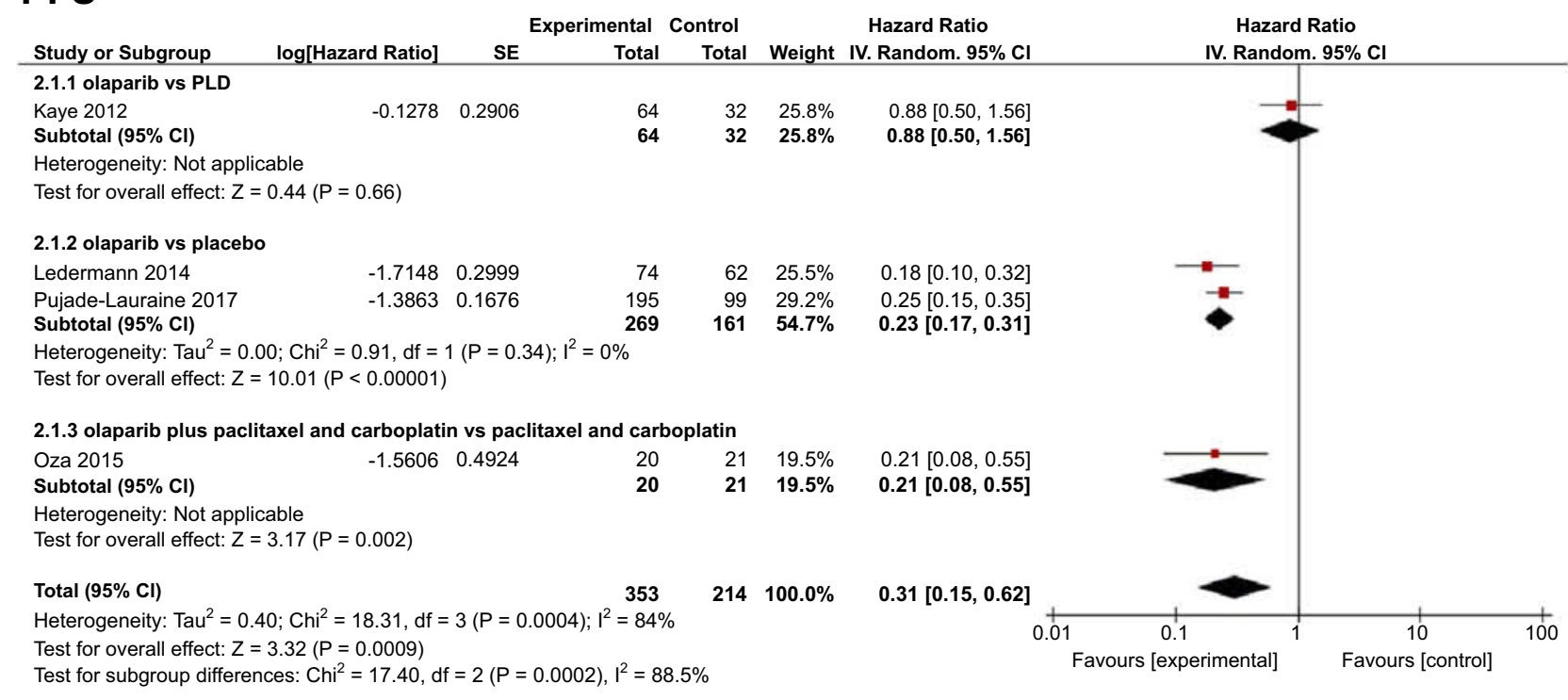

\section{OS}

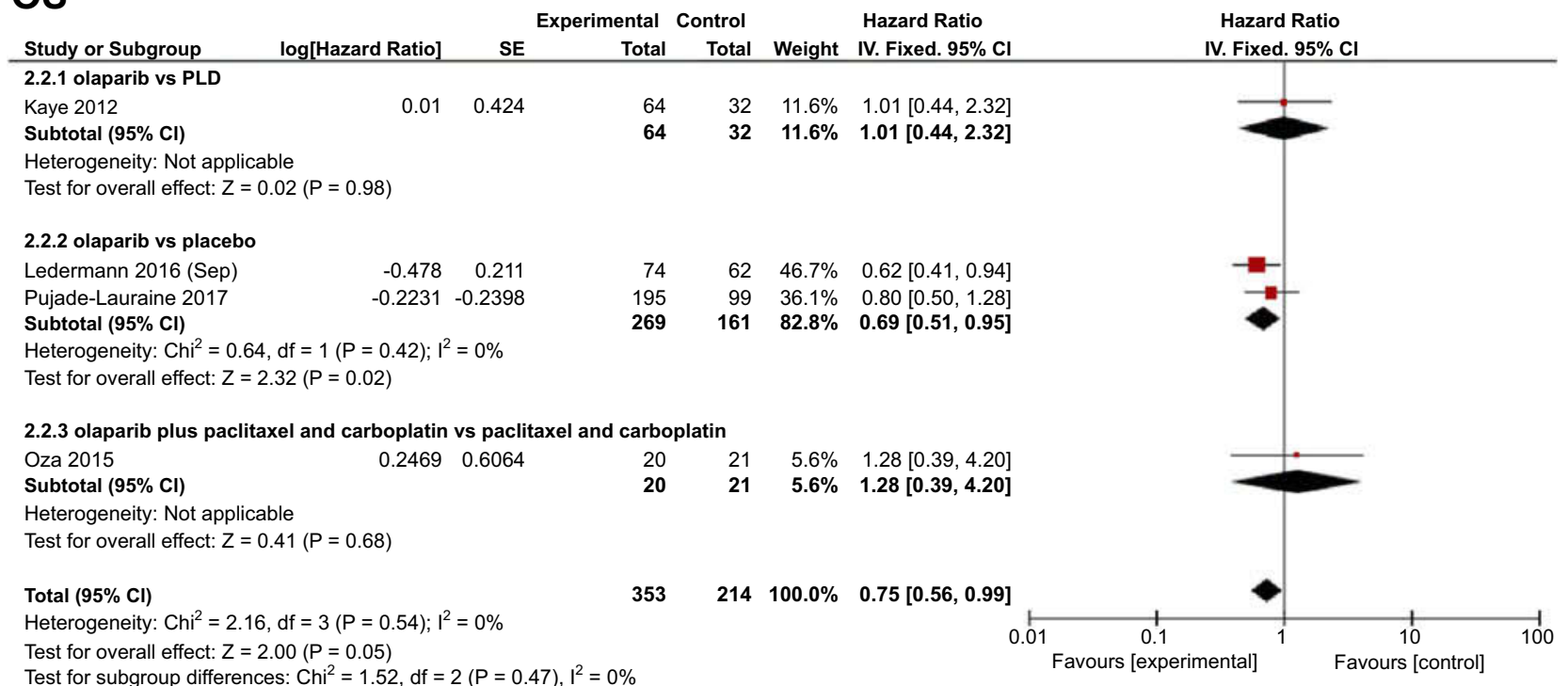

Figure 3 Forest plot of the $\mathrm{HR}$ and $95 \% \mathrm{Cl}$ on the progression-free survival (PFS) and overall survival (OS).

As the validated measure of quality of life, the Functional Assessment of Cancer Therapy-Ovarian Cancer (FACT-O) questionnaires were collected from baseline to disease progression. Two trials found no statistical difference in the total FACT-O score between olaparib and placebo groups (Ledermann 2016 [November], OR=1.38, 95\% $\mathrm{CI}=0.58-3.39, \quad P=0.47$; Friedlander 2018, mean -0.03 points, $P=0.98$ ). In another trial (Kaye 2012), a higher improvement was noted for olaparib compared with PLD for the total FACT-O score $(\mathrm{OR}=7.23,95 \% \mathrm{CI}=1.09$ 143.3, $P=0.039$ ). We analyzed the quality-of-life data from the three trials and calculated the combination of $P$ value by Fisher test using STATA 11.0. The value of
Chi-square was 12.20 , and the combination of $P$-value was 0.058 . The results indicated no appreciable difference in the quality of life for patients receiving olaparib compared with placebo or PLD.

\section{Adverse events}

As there are various adverse events caused by olaparib and the other different chemotherapy drugs (PLD and paclitaxel plus carboplatin), we examined the seven most common adverse events including anemia, fatigue, vomiting, diarrhea, nausea, constipation, and abdominal pain. The adverse events with grade 1-4 were evaluated according to common terminology criteria for adverse events. Overall 
summary statistics showed the increased incidence in five adverse events (anemia, fatigue, diarrhea, nausea, and vomiting) between olaparib and the comparator drugs.

\section{Anemia}

In three trials with 526 participants (Figure 4), olaparib increased the overall risk to suffer low-grade and highgrade anemia compared with other interventions (grade 12: $\mathrm{RR}=4.42,95 \% \mathrm{CI}=2.27-8.60, P<0.0001$; grade $3-4$ : $\mathrm{RR}=7.63,95 \% \mathrm{CI}=2.57-22.67, \quad P=0.0003)$. And we assessed the quality of this evidence to be high in anemia. There was a lower heterogeneity in anemia (grade 1-2: $P=0.92, \mathrm{I}^{2}=0 \%$; grade 3-4: $P=0.91, \mathrm{I}^{2}=0 \%$ ) among the two subgroups. The subgroup analysis indicated that there was no significant difference in the occurrence of anemia between olaparib and PLD groups, while olaparib caused a risen accidence of anemia compared with placebo.

\section{Fatigue}

In overall analysis involving 526 patients, the occurrence of low-grade fatigue was increased by olaparib compared with other interventions (grade 1-2: $\mathrm{RR}=1.49$, 95\% $\mathrm{CI}=1.21$ to $1.84, P=0.0002$, fixed-effect model), while high-grade fatigue induced by olaparib was similar with other interventions (grade 3-4: $\mathrm{RR}=1.63$, 95\% CI $=0.67-$ 3.98, $P=0.28$, fixed-effect model, Figure 4). The subgroup analysis showed a significant difference between olaparib and placebo groups. The results of the meta-analysis presented lower and moderate heterogeneity (grade 1-2: $P=0.47, \mathrm{I}^{2}=0 \%$; grade $3-4: P=0.15, \mathrm{I}^{2}=51.6 \%$ ). And we assessed the quality of this evidence to be moderate.

\section{Vomiting}

Three trials were enrolled to evaluate the risk of vomiting including 526 patients with $B R C A$-mutated ovarian cancer (Figure 5). For overall analysis, low-grade vomiting occurred more frequently (grade 1-2: $\mathrm{RR}=2.08$, 95\% $\mathrm{CI}=1.48-2.93, P<0.0001$, fixed-effect model) and highgrade vomiting presented no difference (grade 3-4: $\mathrm{RR}=1.97, \quad 95 \% \quad \mathrm{CI}=0.50-7.80, \quad P=0.33$, fixed-effect model) in olaparib treatment compared with other interventions. For subgroup analysis, an increased risk of lowgrade vomiting occurred in olaparib groups compared to placebo groups (grade $1-2$ : $\mathrm{RR}=2.34,95 \% \mathrm{CI}=1.56-3.51$, $P<0.0001)$. No differences in the risk of suffering vomiting were found between olaparib and other chemotherapy drugs. The results showed lower heterogeneity (grade 1-2: $P=0.21, \mathrm{I}^{2}=37.4 \%$; grade $\left.3-4: P=0.28, \mathrm{I}^{2}=15.6 \%\right)$. And we assessed the quality of this evidence to be high in lowgrade vomiting and moderate in high-grade vomiting.

\section{Diarrhea}

In overall analysis, the risk of suffering low-grade (grade $1-2)$ diarrhea was increased $(\mathrm{RR}=1.46,95 \% \mathrm{CI}=1.06-$ 2.03, $P=0.02$, fixed-effect model), while the diarrhea high-grade (grade 3-4) showed no difference with olaparib vs other interventions in three trials $(\mathrm{RR}=0.76,95 \%$ $\mathrm{CI}=0.21-2.71, P=0.67$, fixed-effect model, $n=526)$. In subgroup analysis, olaparib increased the incidence rate of low-grade diarrhea in ovarian cancer patients when compared with placebo $(\mathrm{RR}=1.56,95 \% \mathrm{CI}=1.08-2.25$, $P=0.02$ ), while olaparib showed no difference in the incidence of high-grade diarrhea compared with other interventions. There was a considerable heterogeneity in grade 3-4 diarrhea $\left(P=0.10, \mathrm{I}^{2}=63.4 \%\right)$ and a lower heterogeneity in grade $1-2$ diarrhea $\left(P=0.43, \mathrm{I}^{2}=0 \%\right)$ between the two subgroups. And we assessed the quality of this evidence to be moderate (Figure 5).

\section{Nausea}

The occurrence of nausea was seen in three trials with olaparib vs placebo or other chemotherapy drugs in 526 ovarian cancer patients (Figure 6). Compared with other interventions, the frequencies of low-grade nausea (random-effect model) were significantly increased in patients who received olaparib treatment $(\mathrm{RR}=1.87,95 \% \mathrm{CI}=1.33-$ 2.63, $P=0.0004)$. And we assessed the quality of this evidence to be moderate. There was a substantial amount of heterogeneity in low-grade nausea (grade $1-2$ : $P=0.02$, $\left.\mathrm{I}^{2}=81.7 \%\right)$ and a lower heterogeneity in high-grade nausea (grade 3-4: $P=0.22, \mathrm{I}^{2}=33.8 \%$ ) between the two subgroups. Compared with placebo, olaparib could cause more low-grade nausea $(\mathrm{RR}=2.21,95 \% \mathrm{CI}=1.75-2.79$, $P<0.00001)$. However, no significant difference in nausea was found between olaparib and other interventions.

\section{Constipation}

Three trials including 526 patients were assessed for the incidence of constipation (Figure 6). As a result, there was no statistically significant difference for olaparib compared with other interventions in overall analysis $(\mathrm{RR}=0.92,95 \%$ $\mathrm{CI}=0.50-1.70, P=0.80$, random-effect model; $\mathrm{RR}=0.43$, 95\% CI $=0.01-13.85, P=0.63$, random-effect model). And we assessed the quality of this evidence to be moderate in 1- to 2-grade constipation and high in 3- to 4-grade constipation. There was a higher heterogeneity in constipation (grade 1-2: $P=0.04, \mathrm{I}^{2}=76.0 \%$; grade $3-4: \quad P=0.10$, 


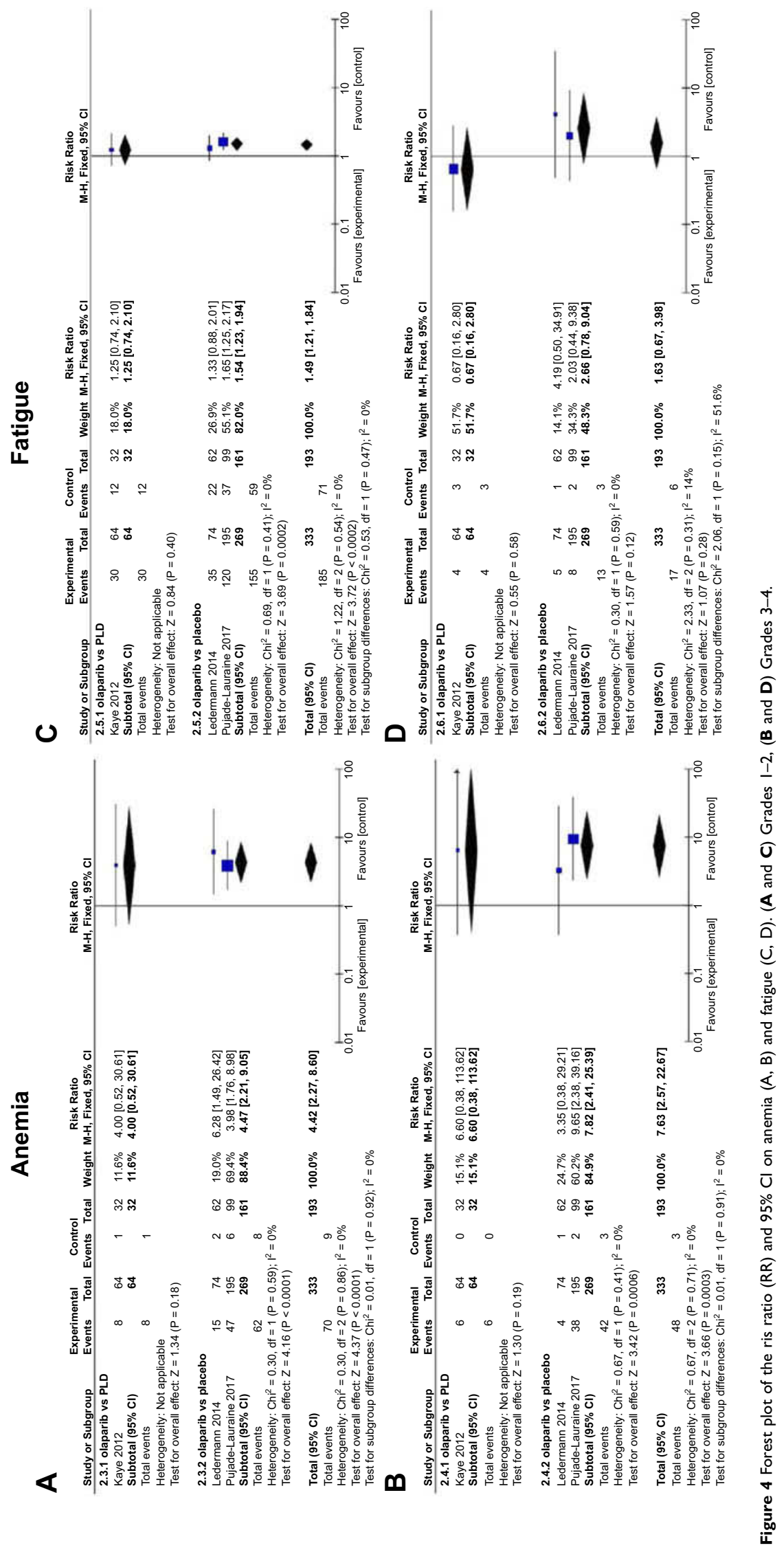



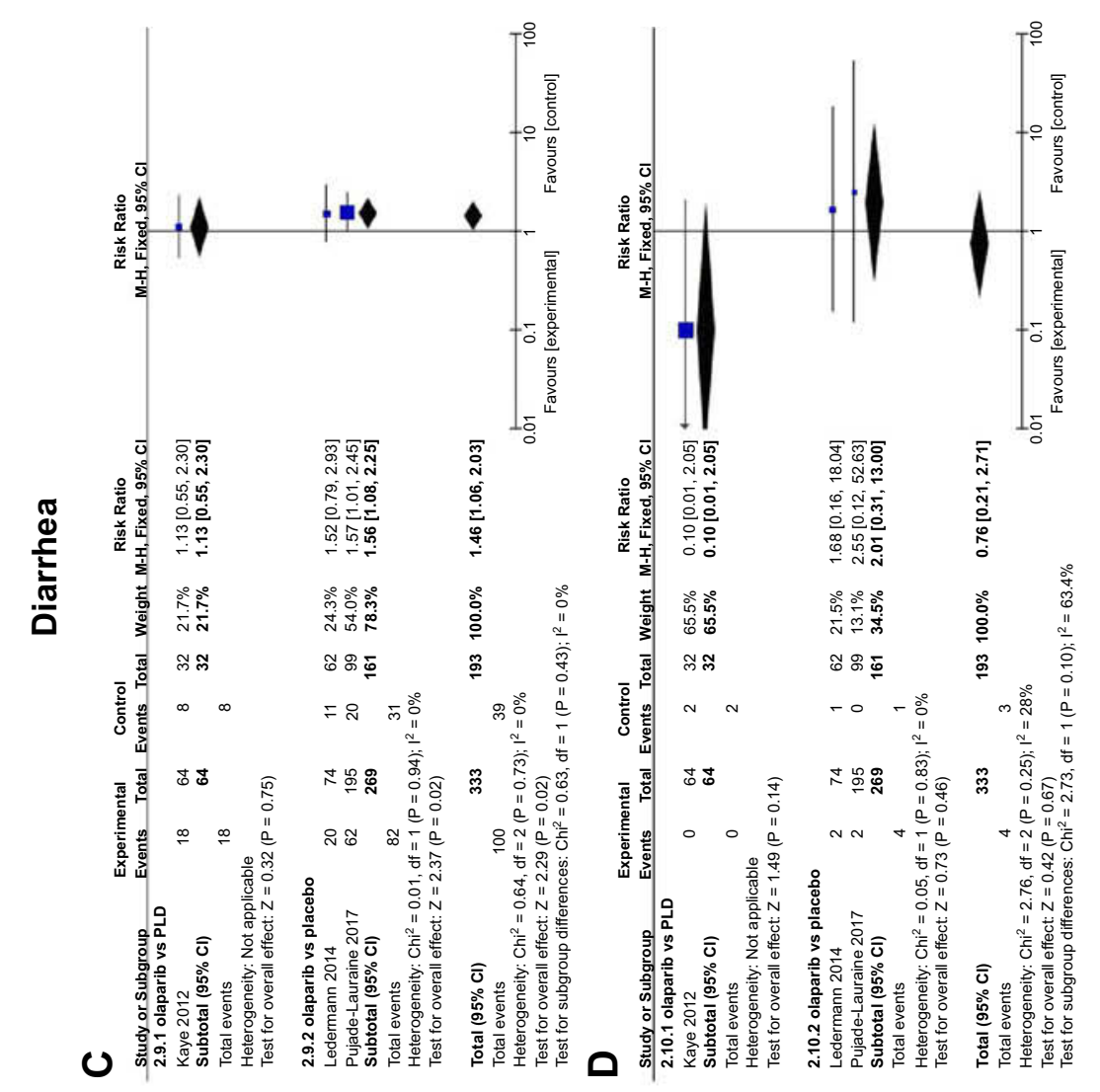

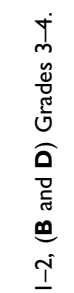

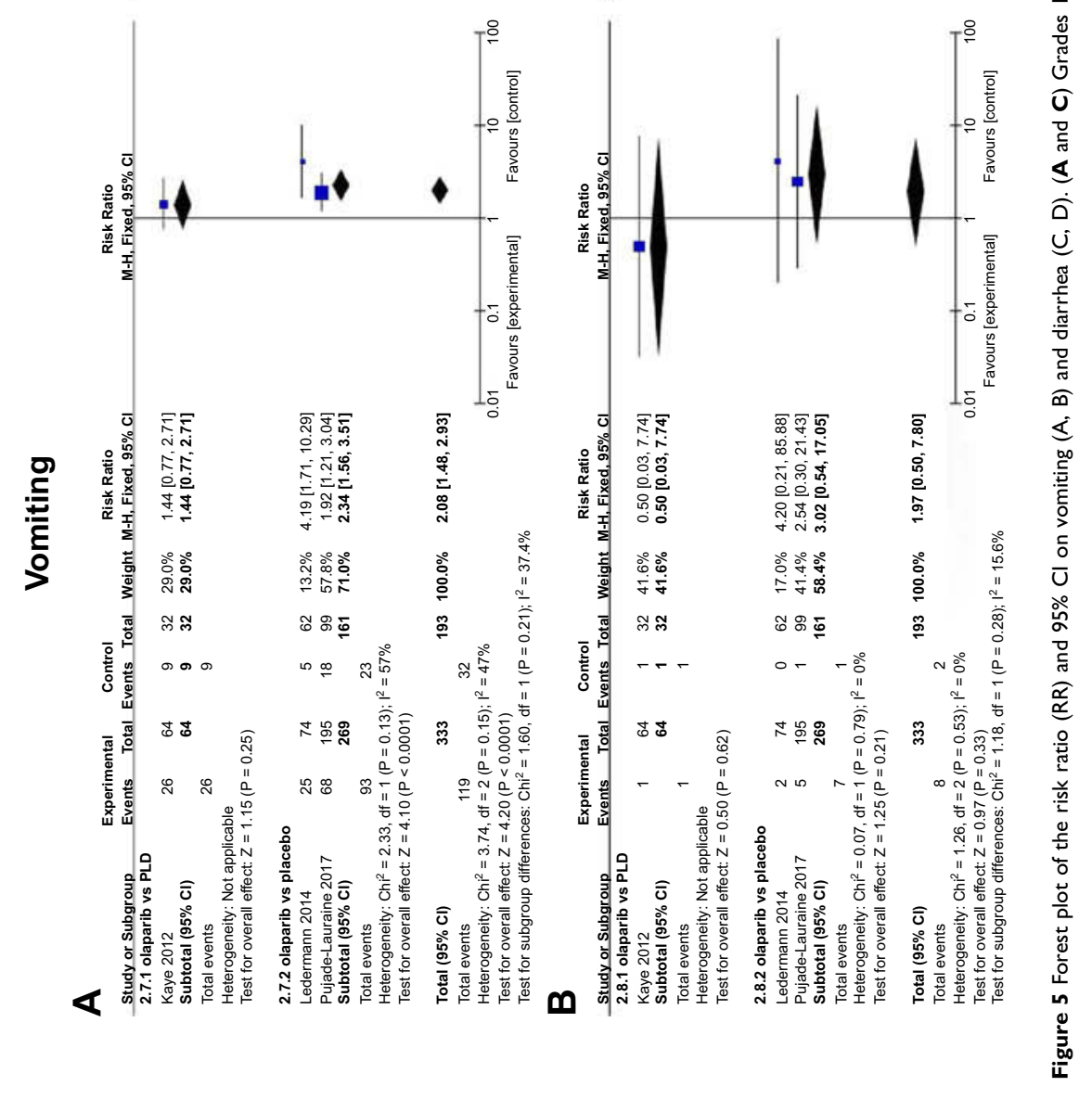



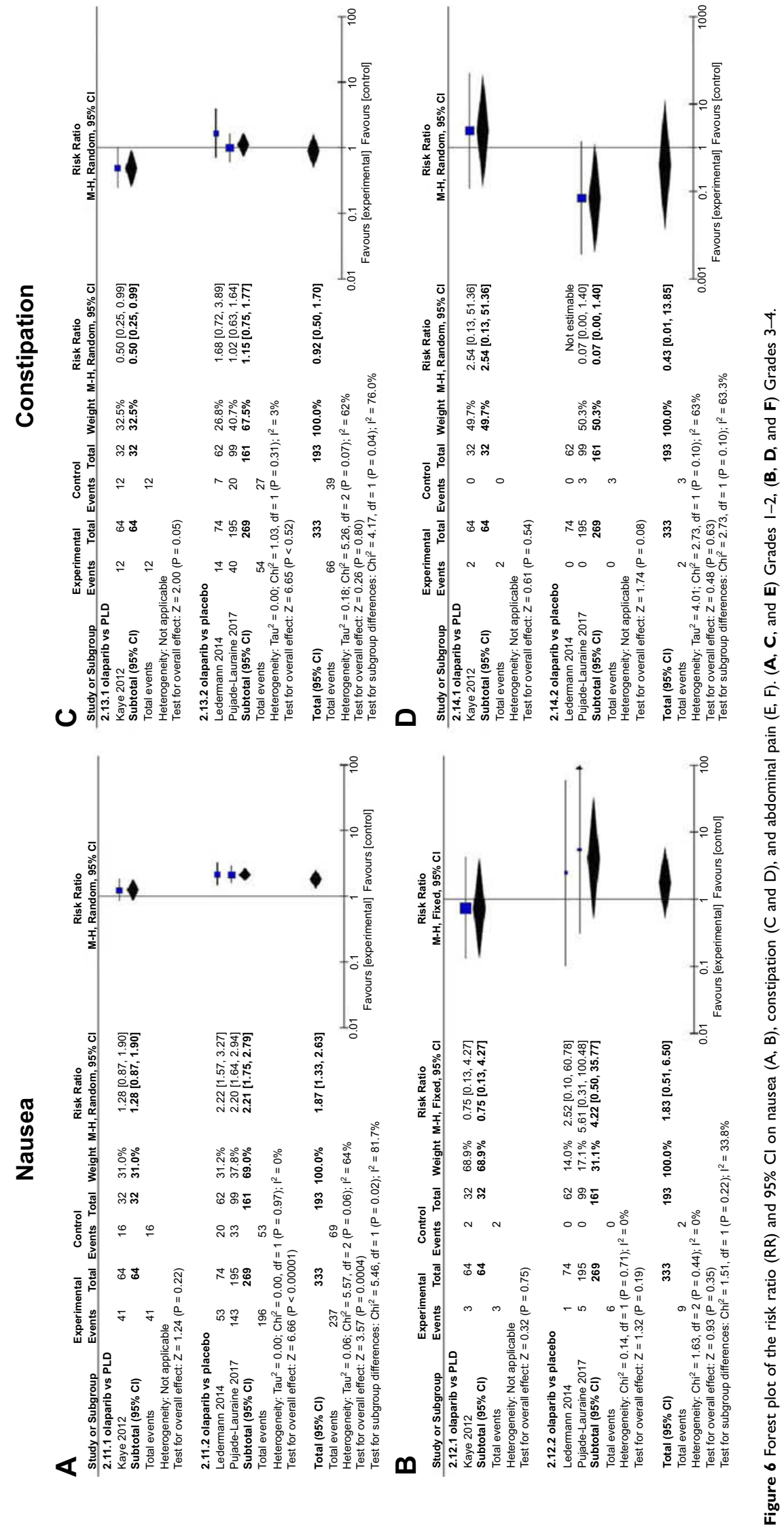


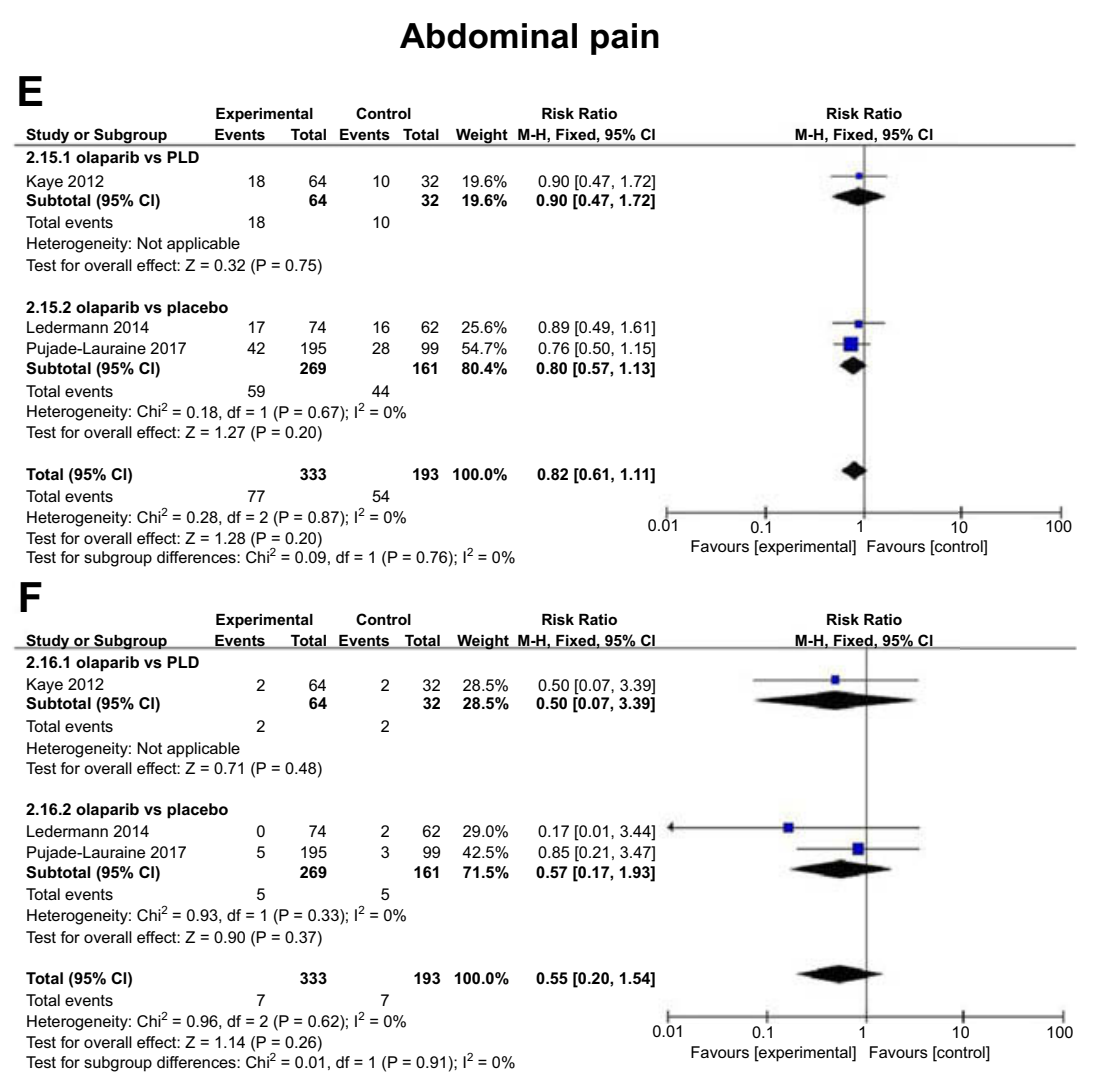

Figure 6 (Continued)

$\mathrm{I}^{2}=63.3 \%$ ) among the two subgroups. In subgroup analysis, olaparib was associated with a lower occurrence of low-grade constipation compared with PLD (grade 1-2: $\mathrm{RR}=0.50,95 \% \mathrm{CI}=0.25-0.99, P=0.05)$.

\section{Abdominal pain}

Three trials evaluated the occurrence of abdominal pain in 526 ovarian cancer patients treated with olaparib or other chemotherapy (Figure 6). Subgroup analyses were noted with lower heterogeneity (grade 1-2: $P=0.76, \mathrm{I}^{2}=0 \%$; grade 3-4: $P=0.91, \mathrm{I}^{2}=0 \%$ ). The overall results showed that the occurrence of abdominal pain in olaparib groups was similar to that in other intervention groups (grade 1-2: $\mathrm{RR}=0.82,95 \% \mathrm{CI}=0.61-1.11, P=0.20$, fixed-effect model; grade 3-4: $\mathrm{RR}=0.55,95 \% \mathrm{CI}=0.20-1.54, P=0.26$, fixedeffect model). And the quality of this evidence was assessed to be moderate.

\section{Discussion}

This review included four studies of RCTs (seven reports) that evaluated the efficacy and safety of olaparib maintenance therapy vs other interventions in women with advanced ovarian cancer. These trials enrolled 567 patients with $B R C A$-mutated recurrent epithelial ovarian, epithelial fallopian tube, or primary peritoneal cancer, which had received platinum-based chemotherapy at least 6 months before entering the trials. Then, the patients were randomized to receive olaparib at a dose of $300 \mathrm{mg}$ or $400 \mathrm{mg}$ twice daily, or olaparib $200 \mathrm{mg}$ twice daily plus other chemotherapy. Treatment continued until disease progression, unacceptable toxicity (adverse events), death, or discontinuation for other reasons. It was noteworthy that a randomized trial (Kaye 2012) compared olaparib doses and found that the $400 \mathrm{mg}$ dose (8.8 months, 95\% $\mathrm{CI}=5.4-9.2$ months) was superior to the $200 \mathrm{mg}$ dose (6.5 months, 95\% CI=5.5 to 10.1 months) for median PFS times. So, $400 \mathrm{mg}$ is possibly the recommended dose. Our analyzing results indicated that olaparib maintenance therapy led to a significantly longer PFS and a slightly better OS in ovarian cancer patients. For the health-related quality of life using FACT-O questionnaires, there were no significant differences in improvement or worsening rates between the olaparib group and other intervention groups. In the included studies, the 
tolerability profile of olaparib treatment was consistent with that reported previously. ${ }^{29-32}$ Compared with placebo, olaparib caused the most common adverse events including diarrhea, nausea, vomiting, anemia, and fatigue at the doses of $300 \mathrm{mg}$ or $400 \mathrm{mg}$ twice per day. However, compared with other chemotherapy drugs, olaparib could not increase the incidences of most adverse events. The meta-analysis results confirmed that olaparib maintenance therapy was generally effective and well tolerated in patients with $B R C A$-mutated advanced ovarian cancer.

This review is applicable to patients with recurrent high-grade $B R C A$-mutated ovarian cancer. As a PARP inhibitor, olaparib is intended for patients with heavily pretreated ovarian cancer that is associated with defective $B R C A$ genes. ${ }^{14}$ The $B R C A$ genes are involved with repairing damaged DNA and normally work to suppress tumor growth. BRCA mutations have an increased risk in the most common type of ovarian cancer. ${ }^{33}$ About $10-15 \%$ of all ovarian cancers are associated with these hereditary $B R C A$ mutations. So, BRCAmutated status in the patients with ovarian cancer was required to detect before olaparib treatment. Compared to the patients with wild-type or unknown $B R C A$ status, the greatest PFS benefit from olaparib maintenance therapy was observed in patients with $B R C A$ mutations. ${ }^{19,20,25,31,32}$ Additionally, preclinical data suggested that olaparib might potentiate the efficacy of DNA-damaging chemotherapies in ovarian cancer, including platinum-containing drugs such as carboplatin. ${ }^{34,35}$ In the patients with platinum-sensitive relapsed serous ovarian cancer, olaparib maintenance treatment significantly improved the duration of PFS compared with placebo. ${ }^{19,20}$ However, retreatment with platinum-based chemotherapy poses the risks including exacerbation of residual neuropathy, enhanced myelosuppression, or onset of hypersensitivity reactions to platinum. ${ }^{36,37}$ Olaparib plus cediranib as maintenance therapy instead of platinum-containing chemotherapy would potentially reduce this risk while prolonging the platinum-free interval. ${ }^{38}$

Using the GRADE system, the available evidence was sufficient for the review authors to make valuable conclusions in assessing the efficacy and safety of olaparib for patients with ovarian cancer (Figure 7). For the outcome of PFS, we did not downgrade the quality of evidence, as although heterogeneity was high $\left(\mathrm{I}^{2}=84 \%\right)$, which might be responsible for different dose levels of olaparib tested, different comparator drugs, olaparib combination therapy vs olaparib monotherapy. For the outcome of OS, we downgraded the quality of evidence to be moderate, as heterogeneity was low $\left(\mathrm{I}^{2}=0 \%\right)$. The outcome adverse events were judged as moderate to high, and $\mathrm{I}^{2}$ ranged from $0 \%$ to $64 \%$.

There were two previous meta-analyses pre-dating our review. Liang 2015 published a meta-analysis that examined three RCTs, ${ }^{19,23,25}$ finding that olaparib maintenance therapy significantly improved PFS (HR $=0.50,95 \%$ $\mathrm{CI}=0.32-0.80$ ), but had no significant advantage on OS $(\mathrm{HR}=0.99,95 \% \mathrm{CI}=0.78-1.25)$ in the patients with recurrent serous ovarian cancer. ${ }^{39}$ In the analyses of toxicity profile, they found the common adverse events including nausea, emesis, abdominal pain, constipation, and anemia with 1 or 2 degrees. Another meta-analysis evaluated four RCTs and mainly tested the therapeutic effect and safety of PARP inhibitors (olaparib, cyclophosphamide, and veliparib) on recurrent epithelial ovarian cancer. ${ }^{17}$ Similarly, olaparib could improve PFS (HR $=0.35,95 \% \mathrm{CI}=0.25$ to 0.49; $\mathrm{HR}=0.42,95 \% \mathrm{CI}=0.29-0.60$ ) but not influence $\mathrm{OS}$ ( $\mathrm{HR}=1.05,95 \% \mathrm{CI}=0.79-1.39$ ), when used as maintenance treatment. In addition, olaparib might cause few severe adverse events (grade 3-4). There is an agreement in the findings of the previous reviews related to the PFS outcome as yet reported, while the outcomes of OS and adverse events in our review are different from these reviews. So our review will be updated when PFS, OS, and toxicity data become available. In addition, compared with Liang 2015, we added the assessments on the bias of risk and the quality of evidence to make the results more accurate.

\section{Conclusion}

As evidenced from our pooled data from 567 patients examined in our review, olaparib maintenance therapy (mostly administered orally at the dose of $400 \mathrm{mg}$ ) was as effective and well tolerated as other therapies with respect to efficacy (mainly measured by PFS, OS, and quality of life) and adverse events. There was high-quality evidence that women with different types of ovarian cancer who received olaparib had significant improvements in PFS. Moreover, we considered that olaparib slightly prolonging OS in patients belonged to moderate-quality evidence. Related to seven adverse events, the evidence ranged from moderate to high quality. Except for the included RCTs in our review, other RCTs about 


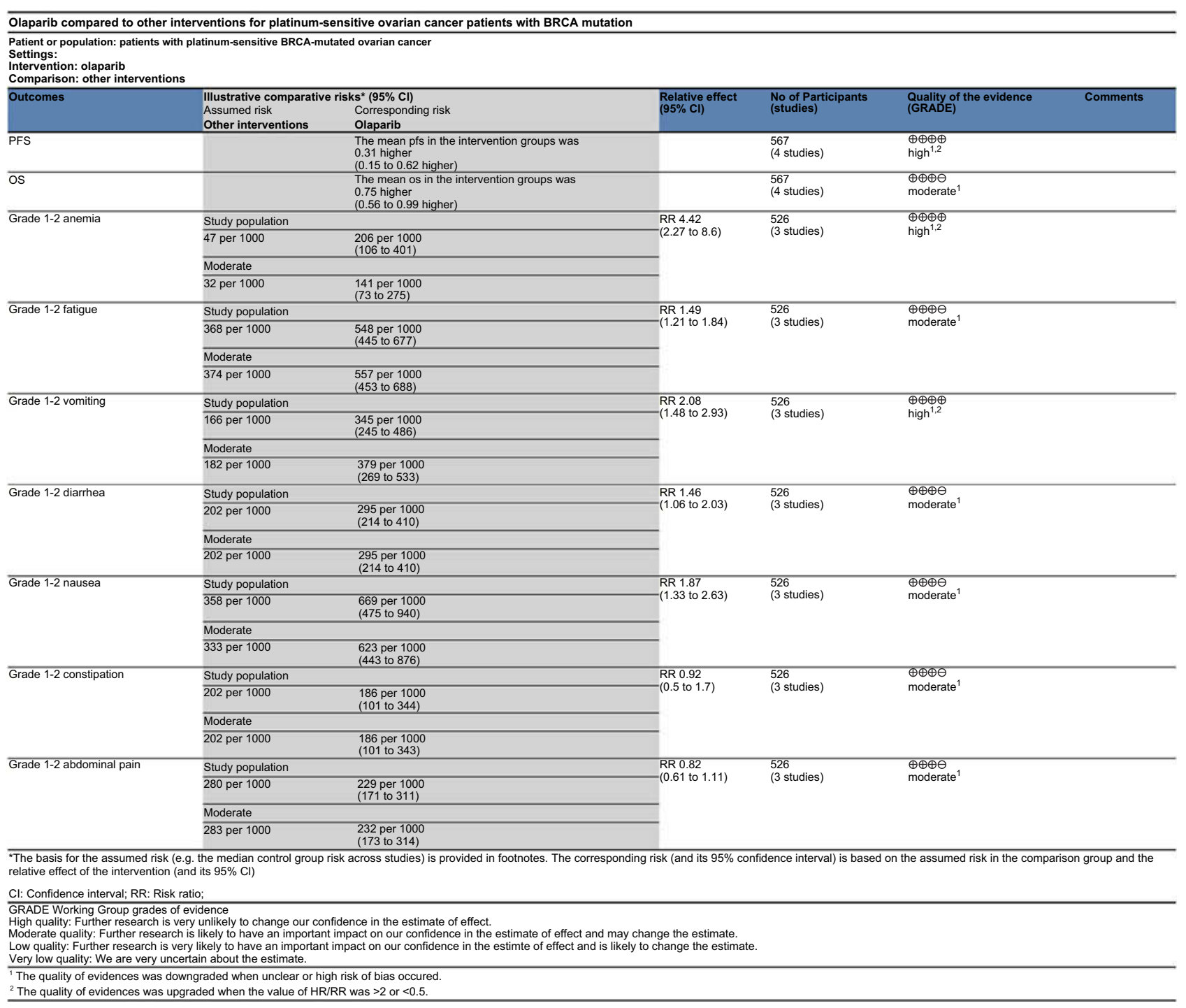

Figure 7 Quality of the evidence.

ovarian cancer patients with olaparib treatment are still ongoing. ${ }^{40-45}$ Therefore, the publication of more survival and safety data from ongoing studies will contribute to future analyses.

Studies powered to detect the outcomes of efficacy and safety in ovarian cancer patients using olaparib maintenance therapy are needed. We await the results of other Phase II and III studies, NCT02392676, NCT03117933, NCT01844986, and NCT02282020, which further assess and update the outcomes including survival, quality of life, and toxicity in ovarian cancer patients with olaparib treatment. ${ }^{42-45}$ Future studies will also compare and analyze the efficacy and toxicity of olaparib at different doses. Additionally, it would be informative to consider also the response to olaparib in patients of wild type or unknown
$B R C A$ status, affected by ovarian cancer, or by different cancers such as prostate cancer that also benefit from olaparib treatment. ${ }^{46}$

\section{Acknowledgment}

This work was supported by National Natural Science Foundation of China (grant number: 81573973).

\section{Author contributions}

All authors contributed to data analysis, drafting and revising the article, gave final approval of the version to be published, and agree to be accountable for all aspects of the work.

\section{Disclosure}

The authors report no conflicts of interest in this work. 


\section{References}

1. Webb PM, Jordan SJ. Epidemiology of epithelial ovarian cancer. Best Pract Res Clin Obstet Gynaecol. 2017;41:3-14.

2. Cancerresearchuk.org. Ovarian cancer statistics. Available from: http://www.cancerresearchuk.org/health-professional/cancer-statis tics/statistics-by-cancer-type/ovarian-cancer. Accessed June 6, 2018.

3. Seer.cancer.gov. Ovarian cancer-cancer stat facts. Available from: https://seer.cancer.gov/statfacts/html/ovary.html. Accessed June 5, 2018

4. Chen WQ, Zheng RS, Baade PD, et al. Cancer statistics in China. $C A$ Cancer J Clin. 2016;66(2):115-132.

5. Fan GY, Wang Y. Clinical analysis of surgical treatment for 105 cases of ovarian cancer. Guide China Med. 2011;9:140-141.

6. Ruan G, Ye L, Liu G, An J, Sehouli J, Sun P. The role of bevacizumab in targeted vascular endothelial growth factor therapy for epithelial ovarian cancer: an updated systematic review and meta-analysis. Onco Targets Ther. 2018;11:521-528.

7. Lo CS, Sanii S, Kroeger DR, et al. Neoadjuvant chemotherapy of ovarian cancer results in three patterns of tumor-infiltrating lymphocyte response with distinct implications for immunotherapy. Clin Cancer Res. 2017;23(4):925-934.

8. Pliarchopoulou K, Pectasides D. Epithelial ovarian cancer: focus on targeted therapy. Crit Rev Oncol Hematol. 2011;79(1):17-23.

9. Menear KA, Adcock C, Boulter R, et al. 4-[3-(4-cyclopropanecarbonylpiperazine-1-carbonyl)-4-fluorobenzyl]-2H-phthalazin-1-one: a novel bioavailable inhibitor of poly (ADP-ribose) polymerase-1. $J$ Med Chem. 2008;51(20):6581-6591.

10. Robson M, Im SA, Senkus E, et al. Olaparib for metastatic breast cancer in patients with a germline BRCA mutation. $N$ Engl $J$ Med. 2017;377(17):523-533. doi:10.1056/NEJMoa1706450

11. Tutt A, Kaufman B, Garber JE, Gelber R, McFadden E, OlympiA: GC. A randomized phase III trial of olaparib as adjuvant therapy in patients with high-risk HER2-negative breast cancer (BC) and a germline BRCA1/2 mutation (gBRCAm). Ann Oncol. 2015;28:67.

12. Yarchoan M, Myzak MC, Johnson BA, et al. Olaparib in combination with irinotecan, cisplatin, and mitomycin $\mathrm{C}$ in patients with advanced pancreatic cancer. Oncotarget. 2017;8(27):44073-44081. doi:10.18632/ oncotarget. 17237

13. Mateo J, Carreira S, Sandhu S, et al. DNA-repair defects and olaparib in metastatic prostate cancer. $N$ Engl J Med. 2015;373(18):16971708. doi:10.1056/NEJMoa1506859

14. Fda.gov. FDA approves Lynparza to treat advanced ovarian cancer. Available from: http://fdaguidance.net/wp-content/uploads/2014/12/ Lynparza.pdf. Accessed July 30, 2018.

15. Fda.gov. FDA approves olaparib tablets for maintenance treatment in ovarian cancer. Available from: https://www.fda.gov/drugs/informatio nondrugs/approveddrugs/ucm572143.htm. Accessed July 30, 2018.

16. Europa.eu. Lynparza: EPAR-Medicine overview. Available from: http://101.96.10.64/www.ema.europa.eu/docs/en_GB/document library/EPAR_-_Summary_for_the_public/human/003726/ WC500180153.pdf. Accessed July 30, 2018.

17. Wiggans AJ, Gemma KSC, Andrew B, Theresa AL, Poly JM. (ADPribose) polymerase (PARP) inhibitors for the treatment of ovarian cancer. Cochrane Database Syst Rev. 2015;5:CD007929. Available from: https://www.cochranelibrary.com/cdsr/doi/10.1002/14651858. CD007929.pub3/full. Accessed July 30, 2018.

18. HemOnc.org. ovarian cancer. Available from: https://hemonc.org/ wiki/Ovarian_cancer\#Olaparib_.28\%20Lynparza.\%2029. Accessed July $30,2018$.

19. Ledermann J, Harter P, Gourley C, et al. Olaparib maintenance therapy in patients with platinum-sensitive relapsed serous ovarian cancer: a preplanned retrospective analysis of outcomes by $B R C A$ status in a randomized phase 2 trial. Lancet Oncol. 2014;15(8):852861. doi:10.1016/S1470-2045(14)70228-1
20. Ledermann J, Harter P, Gourley C, et al. Overall survival in patients with platinum-sensitive recurrent serous ovarian cancer receiving olaparib maintenance monotherapy: an updated analysis from a randomized, placebo-controlled, double-blind, phase 2 trial. Lancet Oncol. 2016;17(11):1579-1589. doi:10.1016/S1470-2045 (16)30376-X

21. Tutt A, Robson M, Garber JE, et al. Oral poly (ADP-ribose) polymerase inhibitor olaparib in patients with BRCA1 or BRCA2 mutations and advanced breast cancer: a proof-of-concept trial. Lancet. 2010;376(9737):235-244. doi:10.1016/S0140-6736(10)60892-6

22. Matulonis UA, Penson RT, Domchek SM, et al. Olaparib monotherapy in patients with advanced relapsed ovarian cancer and a germline BRCA1/2 mutation: a multi study analysis of response rates and safety. Ann Oncol. 2016;27(6):1013-1019. doi:10.1093/annonc/mdw133

23. Kaye SB, Lubinski J, Matulonis U, et al. Phase II, open-label, randomized, multicenter study comparing the efficacy and safety of olaparib, a poly (ADP-ribose) polymerase inhibitor, and pegylated liposomal doxorubicin in patients with BRCA1 or BRCA2 mutations and recurrent ovarian cancer. J Clin Oncol. 2012;30(4):372-379. doi:10.1200/JCO.2011.36.9215

24. Ledermann J, Harter P, Gourley C, et al. Quality of life during olaparib maintenance therapy in platinum-sensitive relapsed serous ovarian cancer. Br J Cancer. 2016;115(11):1313-1320. doi:10.1038/ bjc. 2016.348

25. Oza AM, Cibula D, Benzaquen AO, et al. Olaparib combined with chemotherapy for recurrent platinum-sensitive ovarian cancer: a randomised phase 2 trial. Lancet Oncol. 2015;16(1):87-97.

26. Pujade-Lauraine E, Ledermann JA, Selle F, et al. Olaparib tablets as maintenance therapy in patients with platinum-sensitive, relapsed ovarian cancer and a BRCA1/2 mutation (SOLO2/ENGOT-Ov21): a double-blind, randomized, placebo-controlled, phase 3 trial. Lancet Oncol. 2017;18(9):1274-1284.

27. Liu JF, Barry WT, Birrer M, et al. Combination cediranib and olaparib versus olaparib alone for women with recurrent platinumsensitive ovarian cancer: a randomised phase 2 study. Lancet Oncol. 2014;15(11):1207-1214.

28. Liu JF, Barry WT, Birrer M, et al. Overall survival and updated progression-free survival results from a randomized phase 2 trial comparing the combination of olaparib and cediranib against olaparib alone in recurrent platinum-sensitive ovarian cancer. J Clin Oncol. 2017;35(15 Suppl):5535.

29. Friedlander M, Gebski V, Gibbs E, et al. Health-related quality of life and patient-centred outcomes with olaparib maintenance after chemotherapy in patients with platinum-sensitive, relapsed ovarian cancer and a BRCA1/2 mutation (SOLO2/ENGOT Ov-21): a placebo-controlled, phase 3 randomised trial. Lancet Oncol. 2018;19(8):1126-1134.

30. Fong PC, Boss DS, Yap TA, et al. Inhibition of poly (ADP-ribose) polymerase in tumors from $B R C A$ mutation carriers. $N$ Engl $J$ Med. 2009;361(2):123-134.

31. Ledermann J, Harter P, Gourley C, et al. Olaparib maintenance therapy in platinum-sensitive relapsed ovarian cancer. $N$ Engl $J$ Med. 2012;366(15):1382-1392.

32. Audeh MW, Carmichael J, Penson RT, et al. Oral poly(ADP-ribose) polymerase inhibitor olaparib in patients with BRCA1 or BRCA2 mutations and recurrent ovarian cancer: a proof-of-concept trial. Lancet. 2010;376(9737):245-251.

33. Risch HA, McLaughlin JR, Cole DE, et al. Population BRCA1 and BRCA2 mutation frequencies and cancer penetrances: a kin-cohort study in Ontario, Canada. J Natl Cancer Inst. 2006;98(23):1694-1706.

34. Evers B, Drost R, Schut E, et al. Selective inhibition of BRCA2deficient mammary tumor cell growth by AZD2281 and cisplatin. Clin Cancer Res. 2008;14(12):3916-3925.

35. Rottenberg S, Jaspers JE, Kersbergen A, et al. High sensitivity of BRCA1-deficient mammary tumors to the PARP inhibitor AZD2281 alone and in combination with platinum drugs. Proc Natl Acad Sci USA. 2008;105(44):17079-17084. 
36. Aghajanian C, Blank SV, Goff BA, et al. OCEANS: a randomized, double-blind, placebo-controlled phase III trial of chemotherapy with or without bevacizumab in patients with platinum-sensitive recurrent epithelial ovarian, primary peritoneal, or fallopian tube cancer. J Clin Oncol. 2012;30(17):20392045.

37. González A. Increasing the chances for platinum-sensitive ovarian cancer patients. Future Oncol. 2013;9(12 Suppl):29-35.

38. Moon DH, Lee JM, Noonan AM, et al. Deleterious BRCA1/2 mutation is an independent risk factor for carboplatin hypersensitivity reactions. Br J Cancer. 2013;109(4):1072-1078.

39. Liang JH, Luo RL. Therapeutic effects and safety of olaparib on treatment of recurrent serosity ovarian cancer: a meta-analysis. $J$ Hubei Med Univ. 2015;34(6):538-547.

40. Liu J. Olaparib or cediranib maleate and olaparib compared with standard platinum-based chemotherapy in treating patients with recurrent platinum-sensitive ovarian, fallopian tube, or primary peritoneal cancer. Available from: https://clinicaltrials.gov/ct2/show/ NCT02446600. NLM identifier: NCT02446600. Accessed July 30, 2018.

41. Lee J. Cediranib maleate and olaparib or standard chemotherapy in treating patients with recurrent platinum-resistant or-refractory ovarian, fallopian tube, or primary peritoneal cancer. Available from: https:/clinicaltrials.gov/ct2/show/NCT02502266. NLM identifier: NCT02502266. Accessed July 30, 2018.
42. Gourley C, Aghajanian C. Olaparib maintenance treatment versus placebo in patients with PSR ovarian cancer who are in CR or PR to platinum-based chemotherapy and whose tumors carry sBRCAm or HRR-associated genes mutations. Available from: https://clinical trials.gov/ct2/show/NCT02392676. NLM identifier: NCT02392676. Accessed July 30, 2018.

43. McGregor N, Vakirtzis T. Olaparib +/- cediranib or chemotherapy in patients with BRCA mutated platinum-resistant ovarian cancer (OCTOVA). Available from: https://www.clinicaltrials.gov/ct2/show/ NCT03117933. NLM identifier: NCT03117933. Accessed July 30, 2018.

44. DiSilvestro P, Moore K. Olaparib maintenance monotherapy in patients with $B R C A$ mutated ovarian cancer following first line platinum based chemotherapy. (SOLO-1). Available from: https://www.clinicaltrials.gov/ ct2/show/NCT01844986. NLM identifier: NCT01844986. Accessed July $30,2018$.

45. Es L. Olaparib treatment in relapsed germline breast cancer susceptibility gene (BRCA) mutated ovarian cancer patients who have progressed at least 6 months after last platinum treatment and have received at least 2 prior platinum treatments (SOLO3). Available from: https://www.clinicaltrials.gov/ct2/show/NCT022820200. NLM identifier: NCT022820200. Accessed July 30, 2018.

46. Morra F, Merolla F, Napolitano V, et al. The combined effect of USP7 inhibitors and PARP inhibitors in hormone-sensitive and castration-resistant prostate cancer cells. Oncotarget. 2017;8(19):3181531829.

\section{Publish your work in this journal}

Cancer Management and Research is an international, peer-reviewed open access journal focusing on cancer research and the optimal use of preventative and integrated treatment interventions to achieve improved outcomes, enhanced survival and quality of life for the cancer patient.
The manuscript management system is completely online and includes a very quick and fair peer-review system, which is all easy to use. Visit http://www.dovepress.com/testimonials.php to read real quotes from published authors. 